\title{
Effect of Using Mouthwash Solution on Commensal Flora of the Oral Cavity among Female Students in Qassim University
}

\author{
Shaden Mohammed Alhathloul, Amal Hussain Mohammed Ali \\ Department of Medical Laboratories, College of Applied Medical Sciences, Qassim University, Qassim, Kingdom of Saudi Arabia \\ Email: Ama.Ali@qu.edu.sa
}

How to cite this paper: Alhathloul, S.M. and Ali, A.H.M. (2020) Effect of Using Mouthwash Solution on Commensal Flora of the Oral Cavity among Female Students in Qassim University. Journal of Biosciences and Medicines, 8, 135-142.

https://doi.org/10.4236/jbm.2020.83012

Received: January 29, 2020

Accepted: March 9, 2020

Published: March 12, 2020

Copyright $\odot 2020$ by author(s) and Scientific Research Publishing Inc. This work is licensed under the Creative Commons Attribution International License (CC BY 4.0).

http://creativecommons.org/licenses/by/4.0/

\begin{abstract}
Oral health is the pathway to one's general health and wellness. Mouthwash is a product used as an adjunct to oral hygiene practices as an antiseptic and antiplaque and antimicrobial agent. The awareness of using mouthwash solution is so limited worldwide. And maybe there's effect of mouthwash on commensal flora. The aim of this study is to measure the effect of using Mouthwash solution on commensal flora of the oral cavity. In this study, we determined three groups, the first group is considered as a basic group where Female students are not using any mouthwash, the second group used mouthwash twice daily for two weeks, group number three used mouthwash twice weekly. We take cytological sample scraping from oral cavity. All the cytological sections of all groups stained with Papanicolaou stain and examined the slides under the microscope. The study came out with the following results: The basic group that did not use any mouthwash remained unchanged, while the second group, who used mouthwash twice daily, showed a clear epithelial tissue for any bacteria and a clear background. Group number three, who used mouthwash twice a week, showed countable bacteria in epithelial tissue with a clear background. Finally, the study concluded: Cosmetic mouthwash solution has a bad effect on commensal flora if you over used it and leads to killing all your normal flora in oral cavity as much as therapeutic mouthwash will do.
\end{abstract}

\section{Keywords}

Mouthwash, Commensal Oral Flora, Papanicolaou Stain, Awareness, Effect

\section{Introduction}

Oral health is the pathway to one's general health and wellness. Many systemic 
diseases show their early signs in the oral cavity. So the Dental health cannot be separated from general health. Mouthwash is a product used as an adjunct to oral hygiene practices as an antiseptic and anti-plaque and anti-microbial agent. The oral environment provides favorable conditions for the proliferation of bacteria, where pathogen infection may cause dental caries by creating an acidic environment and biofilm around teeth and gums. Numerous types of bacteria are associated with tooth decay, and the most prominent one among these is Streptococcus mutans. Oral bacteria include streptococci, lactobacilli, staphylococci and corynebacteria, with a great number of anaerobes, especially bacteroides [1].

Mouthwash is applied to teeth to prevent cavities, fluoride is a mineral that works by making the teeth more resistant and stronger to decay caused by acid and bacteria [2] [3]. The oral mucosal epithelium is a barrier that separates the underlying tissues from their environment. It consists of two layers, the surface stratified squamous epithelium and the deeper lamina propria [4]. Basically, the use of mouthwash does not eliminate the need for both tooth brush and flossing [5]. The Food and Drug Administration classifies mouthwash as either cosmetic or therapeutic [6]. The common use involves rinsing the mouth with about 10 $15 \mathrm{ml}$ of mouthwash two times a day after brushing [7].

Mouthwash is not recommended for children younger than 6 years of age. Swallowing reflexes may not be well developed in children and may be swallowing large amounts of the mouthwash, which can trigger adverse events like nausea, vomiting, and intoxication (due to the alcohol content in some rinses) [8].

Unfortunately, the awareness of using mouthwash solution is so limited worldwide. And maybe there's effect of mouthwash on commensal oral flora. Therefore, the aim of this study is to measure the effect of using Mouthwash solution on commensal flora of the oral cavity among Female students in Qassim University. By using Papanicolaoustain as a cytological technique to determine the cellular change.

\section{Methodology}

The design of this study is an experimental study to evaluate the effect of mouthwash solution on commensal bacteria of the oral cavity among Female students in Qassim University. The sample obtained from Female students in Qassim University and stained with Papanicolaou stain. The study conducted from September, 2019 to November, 2019. The sample includes Female students in Qassim University, and the sample size is 25 students.

The basic control group includes students before using the mouthwash solution, then students subdivided into three group, group number one is students did not use mouthwash, the second group students are used the mouthwash twice daily. Whereas, the group number three used the mouthwash twice per week. After two weeks the second sample collected to compare the differentness between the second and third groups and to see the effectiveness of using mouthwash solution on commensal flora of the oral cavity among female students in Qassim University. 


\section{Effect}

Will define as the effect of mouthwash solution on the commensal flora of the oral cavity among Female students in Qassim University.

\section{Results}

The students subdivided into three group, group number one is basic group of students who are not using mouthwash, the second group students are used the mouthwash twice daily. Whereas, the group number three used the mouthwash twice per week. After two weeks the second sample collected to compare the differentness between the second and third groups and to see the effectiveness of using mouthwash solution on commensal flora of the oral cavity.

Group (1) "Basic":

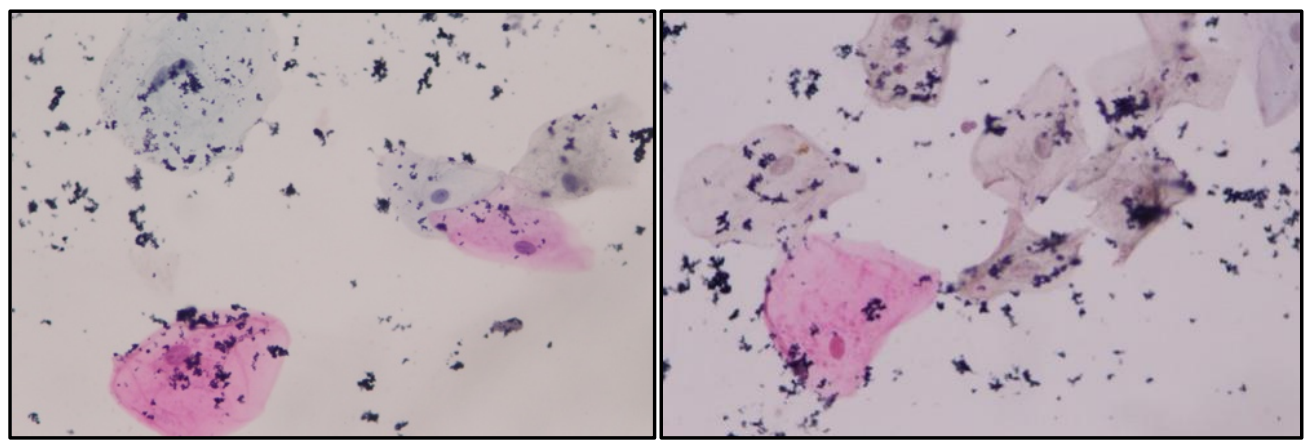

The basic group that did not use any mouthwash their result remained unchanged.

The amount of bacteria in the first picture is the same as it shows in the second picture which is taken after two weeks as the participant did not use any mouthwash solution.

Group (2) "Twice daily":

Sample (1):

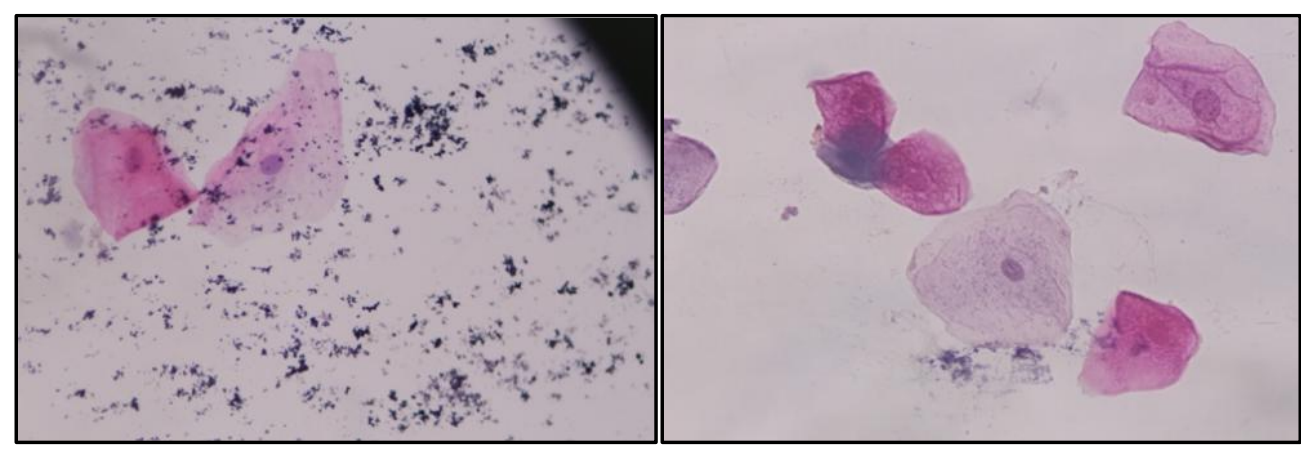

The first picture shows a huge amount of bacteria out and inside the epithelial tissue. After two weeks of daily using mouthwash the epithelial shows very clear of bacteria.

Sample (2): 


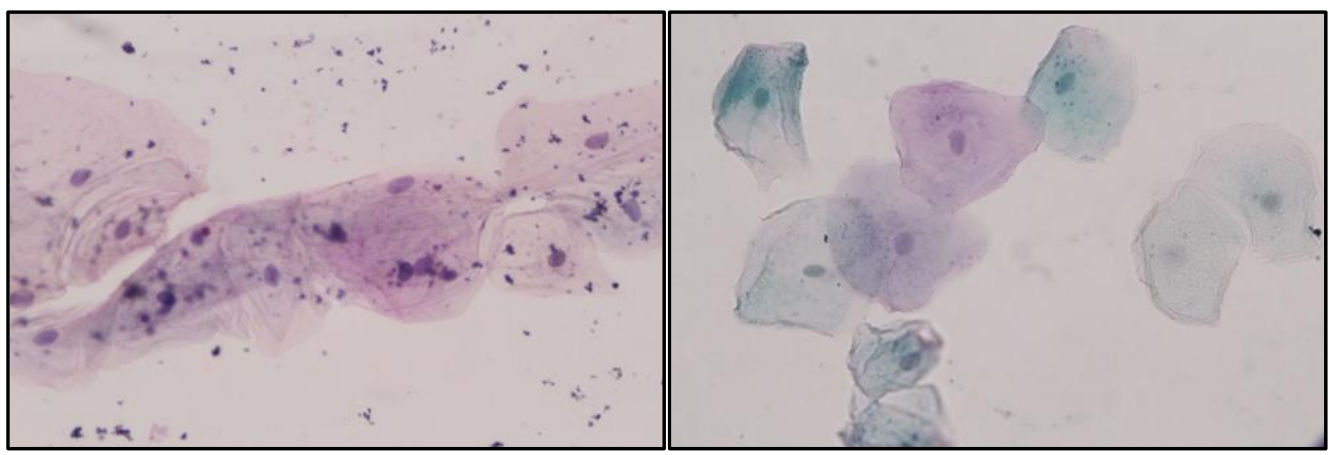

The first picture shows a lot of bacteria out and inside the epithelial tissue. The second picture shows clear epithelial tissue of any bacteria.

Sample (3):

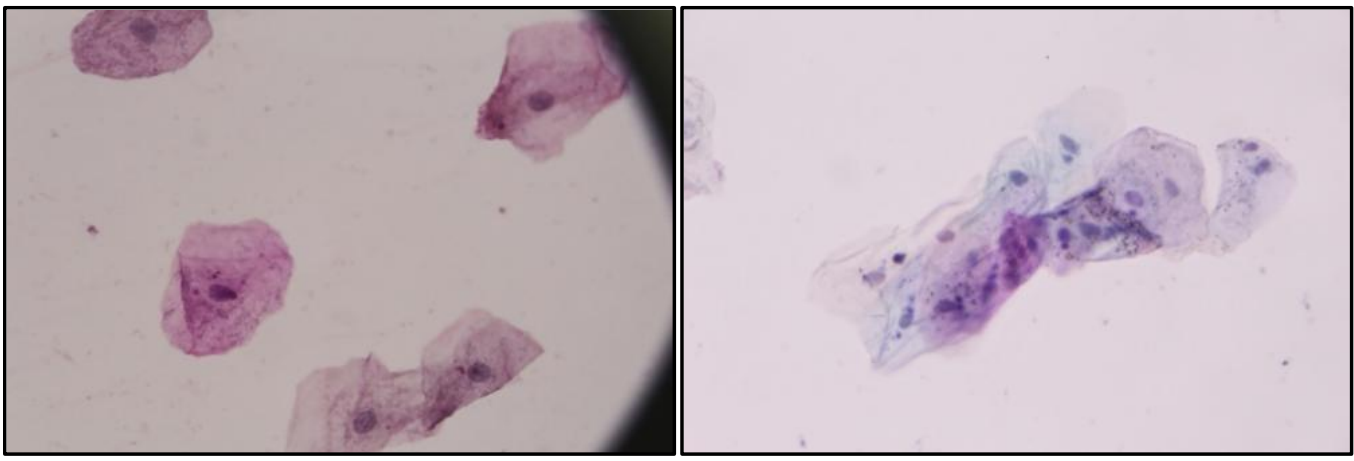

The participant already uses mouthwash but not daily, so the first picture shows a little amount of bacteria out and inside whereas the second picture after she used the mouthwash daily there's few bacteria inside the epithelial with clear background.

Sample (4):

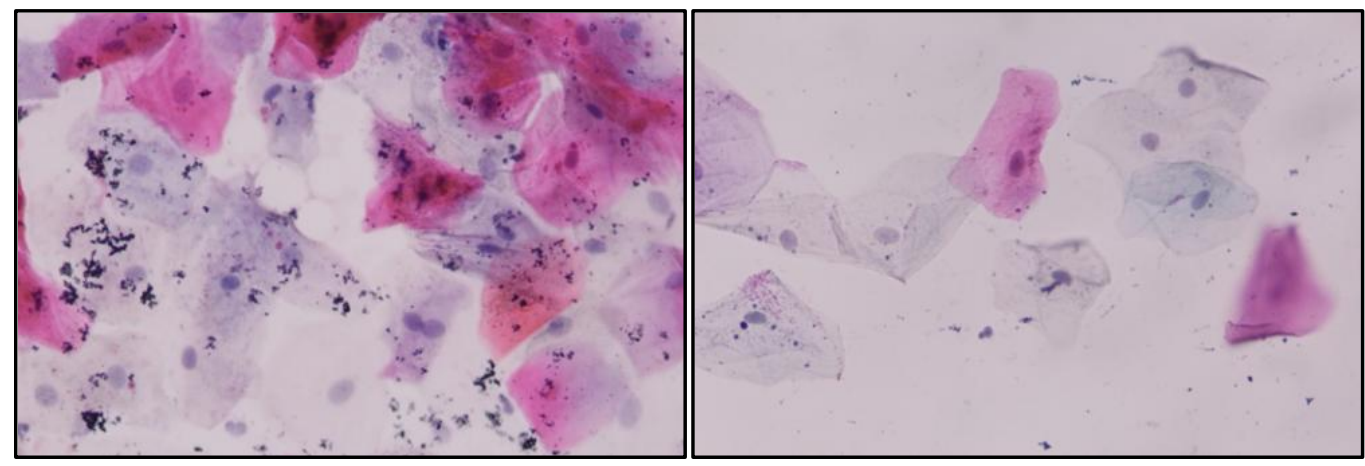

The first picture before using any mouthwash shows a great amount of bacteria out and inside the epithelial tissue, after using the mouthwash daily for two weeks the bacteria amount decreased in significant way even though there was a little bacteria inside the epithelial tissue.

Sample (5): 


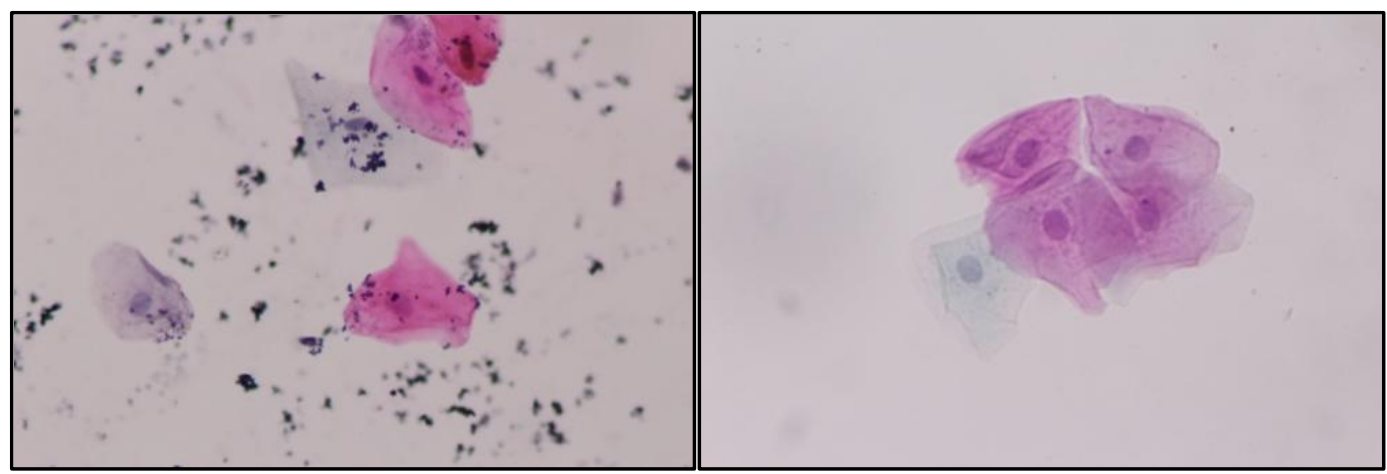

The first picture shows a great amount of bacteria outside the epithelial with a little amount inside. The second picture shows epithelial tissue without bacteria either inside or outside.

Group (3) “Twice weekly":

Sample (1):

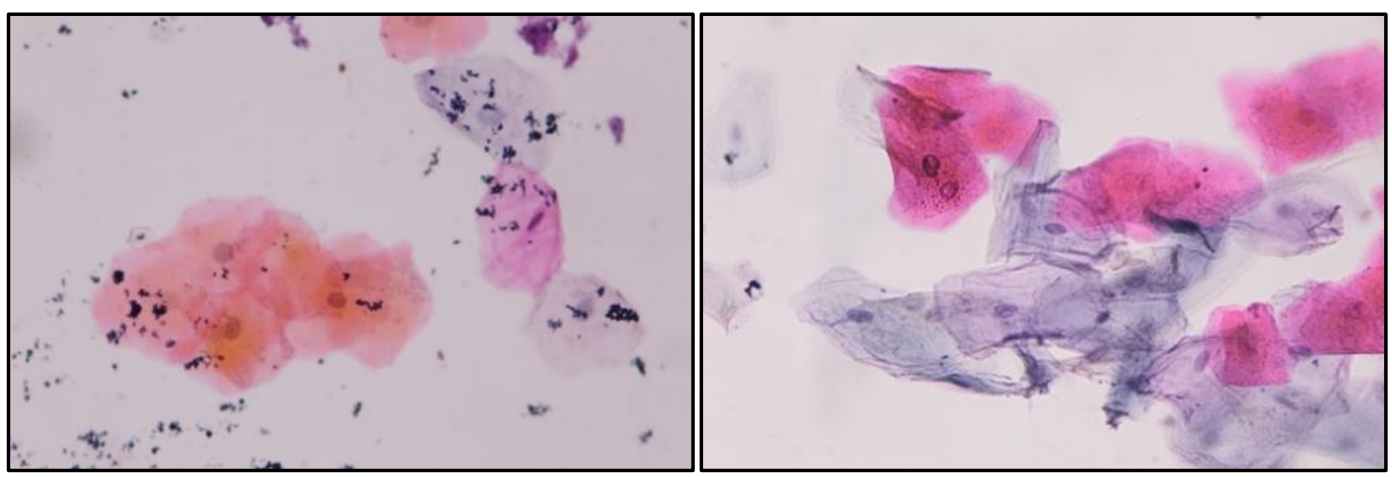

The first picture shows a considerable amount of bacteria out and inside the epithelial tissue, the second picture shows few bacteria inside the epithelial.

Sample (2):

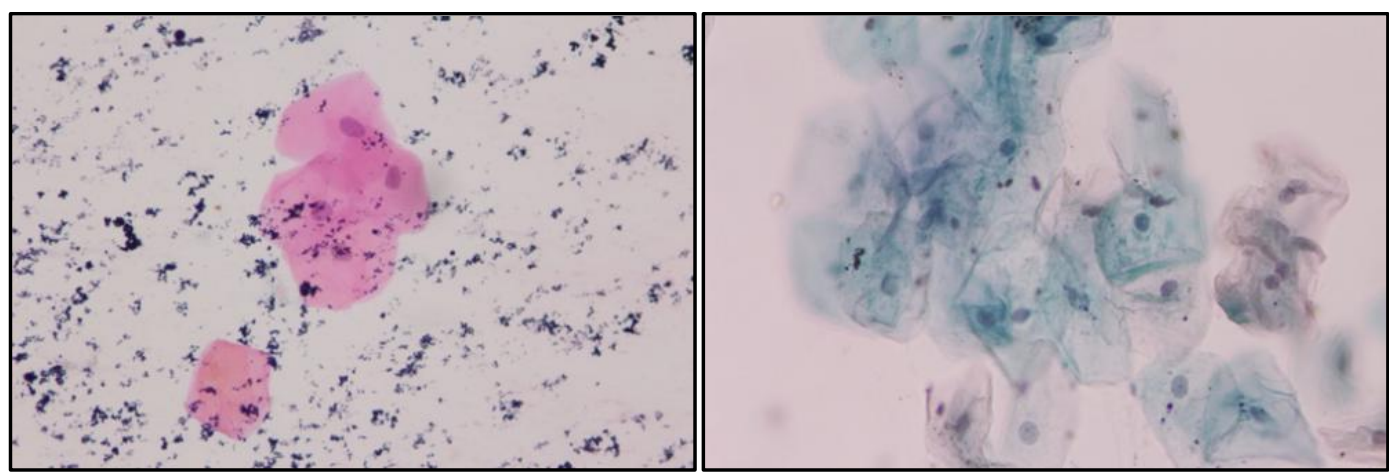

The first picture shows a great amount of bacteria out and inside the epithelial tissue, after two weeks of using the mouthwash twice weekly the second picture shows little bacteria inside the epithelial tissue with clear background.

Sample (3): 


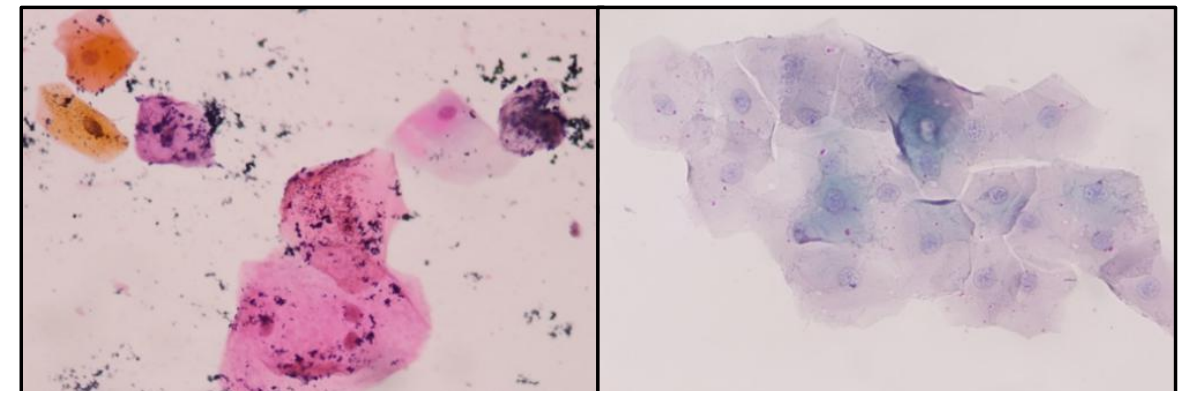

In the first picture, there's a great amount of bacteria inside and outside the epithelial tissue, whereas the second picture shows barely bacteria inside the epithelial tissue with clear background.

Sample (4):

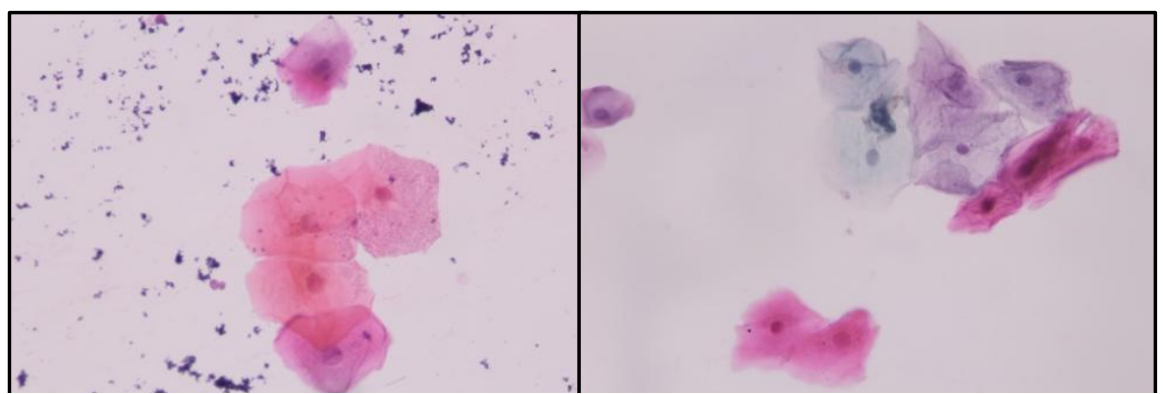

The first picture shows a great amount of bacteria outside the epithelial tissue, after using mouthwash the second picture shows clear background of any bacteria.

Sample (5):

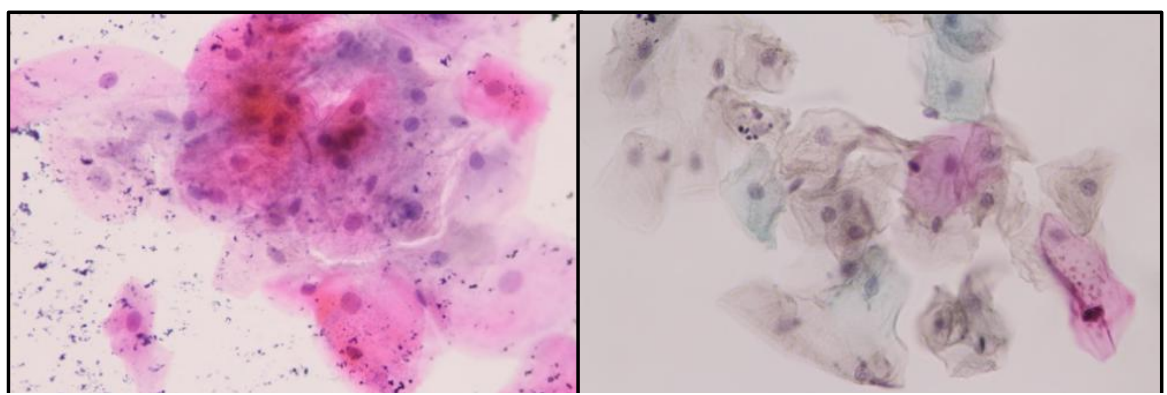

In the first picture before using mouthwash there's a lot of bacteria inside and outside the epithelial tissue, after two weeks of using the mouthwash twice per week the epithelial tissue appeared very clear of any bacteria.

\section{Discussion}

In the current study, while in group number two the epithelial tissue shows clean from bacteria also the background was clear, which indicates the mouthwash has affected the bacteria after two weeks of twice daily using the mouthwash. In group number three where the students used the mouthwash twice weekly the 
epithelial tissue appeared with countable bacteria inside and clear outside the epithelial tissue.

The research supports an Australian study in 2009, which said the mouthwashes containing alcohol may have opposite effect, as they allow carcinogens to penetrate the lining of the mouth more easily and thus contribute to an increased incidence of disease [9].

The study of Researchers at Harvard University found that people who use mouthwash more than once a day can lead to diabetes or a dangerous rise in blood sugar, within three years. Kaumudi Joshipura, professor of epidemiology at Harvard School of Public Health, said: "Most of these antibacterial ingredients in mouthwash are not selective [10]. As long as over use of mouthwash may lead to developing diabetes or dangerous rise in blood sugar with long term of using it will affect your oral health as well. This proves not only therapeutic mouthwash should be used carefully, also cosmetics mouthwash should be used in a balanced way that won't cause harm.

On another vision there's another study found that mouthwash has strong effect on blood-pressure. This study has done in Centre of Genomic Regulation in Barcelona (Gabaldon's lab), Spain which found that people who rinsed their mouths with antibacterial mouthwash before physical activity showing high blood pressure under resting conditions, so this showed the mouthwash impact on the effects of exercise. The bad effect on the oral bacteria leads to complicated impact of cardiovascular health [11]. Our result is compatible with this study, which shows the bad effect of mouthwash in human health by killing commensal flora of the oral cavity.

\section{Conclusion}

Cosmetic mouthwash solution has some bad effects on commensal oral flora if you over used it and may lead to killing all your normal flora in the oral cavity as much as therapeutic mouthwash will do. This proves that the optimal way to use the mouthwash Solution is to use it in balanced manner that won't cause an opposite effect.

\section{Conflicts of Interest}

The authors declare no conflicts of interest regarding the publication of this paper.

\section{References}

[1] Sn, B. (2016) Knowledge, Attitude and Use of Mouthwash among Dental and Medical Students of the University of Nairobi. International Journal of Dentistry and Oral Health, 2, 2-6. https://doi.org/10.16966/2378-7090.198

[2] Papagerakis, S., Pannone, G., Zheng, L., About, I., Taqi, N., Nguyen, N.P.T., Papagerakis, P., et al. (2014) Oral Epithelial Stem Cells-Implications in Normal Development and Cancer Metastasis. Experimental Cell Research, 325, 111-129. https://www.ncbi.nlm.nih.gov/pmc/articles/PMC4157336/ 
https://doi.org/10.1016/j.yexcr.2014.04.021

[3] Ciancio, S.G. (1990) Dental Products. In: Swarbrick, J. and Boylan, J.C., Eds., Encyclopedia of Pharmaceutical Technology, Volume 3, Marcel Dekker, Inc., New York and Basel, 425-456.

[4] Danielsen, B., Baelum, V. and Manji, F. (1997) Mouthwash Effectiveness Assessed. The Journal of the American Dental Association, 128, 1220. https://doi.org/10.14219/jada.archive.1997.0395

[5] Uitenbroek, D.G., Schaub, R.M., Tromp, J.A. and Kant, J.H. (1989) Dental Hygienists' Influence on the Patients' Knowledge, Motivation, Self-Care, and Perception of Change. Community Dentistry and Oral Epidemiology, 17, 87-90.

https://www.ncbi.nlm.nih.gov/pubmed/2920545 https://doi.org/10.1111/j.1600-0528.1989.tb00595.x

[6] Zero, D.T. (2006). Dentifrices, Mouthwashes, and Remineralization/Caries Arrestment Strategies. https://www.ncbi.nlm.nih.gov/pmc/articles/PMC2147065

[7] Yang, S.-J., Han, S.-H., Lee, A.-R., et al. (2015) Evaluation of Antimicrobial Effects of Commercial Mouthwashes Utilized in South Korea. BMB Reports, 48, 42-47.

https://www.ncbi.nlm.nih.gov/pmc/articles/PMC4345641 https://doi.org/10.5483/BMBRep.2015.48.1.090

[8] Mouthwash and What the Different Types Can Do for You (2018). https://www.gentledentalcenter.com/different-types-mouthwash/

[9] McCullough, M.J. and Farah, C.S. (2008) The Role of Alcohol in Oral Carcinogenesis with Particular Reference to Alcohol-Containing Mouthwashes. Australian Dental Journal, 53, 302-305. https://doi.org/10.1111/j.1834-7819.2008.00070.x

[10] Knapton, S. (2017) Mouthwash May Kill Beneficial Bacteria in Mouth and Trigger Diabetes, Harvard Study Suggests.

https://www.telegraph.co.uk/science/2017/11/22/mouthwash-may-kill-beneficial-ba cteria-mouth-trigger-diabetes/

[11] Cutler, C., Kiernan, M., Willis, J.R., Gallardo-Alfaro, L., Casas-Agustench, P., White, D., Hickson, M., Gabaldon, T. and Bescos, R. (2019) Post-Exercise Hypotension and Skeletal Muscle Oxygenation Is Regulated by Nitrate-Reducing Activity of Oral Bacteria. Free Radical Biology and Medicine, 143, 252-259.

https://doi.org/10.1016/j.freeradbiomed.2019.07.035 